PoS $\quad \begin{aligned} & \text { PROCEEDINGS } \\ & \text { OF SCIENCE }\end{aligned}$

\title{
Dark Matter Searches with the CMS Experiment
}

\section{Guillelmo Gómez-Ceballos* on behalf of the CMS Collaboration}

Massachusetts Institute of Technology

E-mail: guillelmo.gomez.ceballos@cern.ch

This conference report briefly reviews the status of the dark matter searches at the CMS experiment with the data collected in 2016 at $\sqrt{s}=13 \mathrm{TeV}$ using a relatively large number of different final states.

2nd World Summit: Exploring the Dark Side of the Universe

25-29 June, 2018

University of Antilles, Pointe-à-Pitre, Guadeloupe, France

${ }^{*}$ Speaker. 


\section{Introduction}

The nature of the dominant matter component in the Universe, so-called dark matter (DM), remains one of the biggest open questions in current particle physics. A compelling hypothesis that accommodates the observed relic abundance of DM is the existence of an electrically neutral and stable particle having weak interactions with the standard model (SM) particles, in addition to gravitational interactions, and a mass in the range of a few $\mathrm{GeV}$ up to several TeV. Such an extension of the SM could result in detectable signatures, and many models predict observable rates for the production of such particles at the Large Hadron Collider.

The DM particle can be detected in a direct production mode, or as a by-product of more complex final states, as seen in Fig. 1. The focus on this report is on the direct production case using the data collected by the CMS experiment [1] in 2016 at $\sqrt{s}=13 \mathrm{TeV}$.

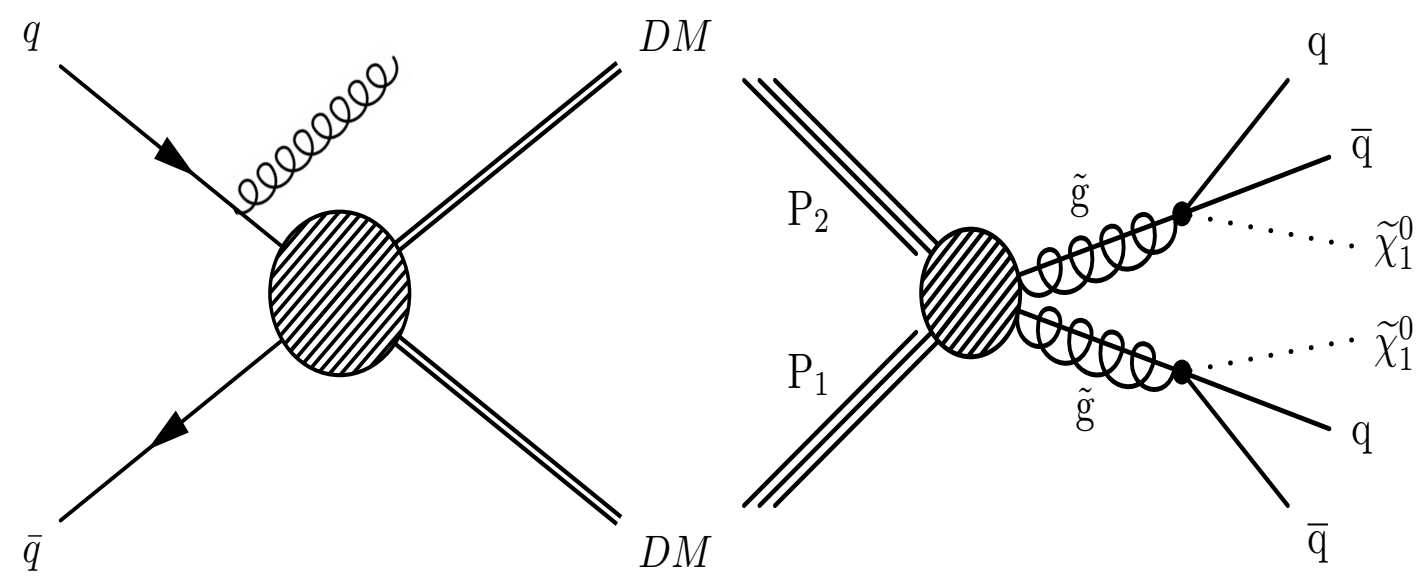

Figure 1: Example of DM particle production as a direct production mode (left) and as a by-product of more complex final states (right).

\section{Experimental Setup}

Most analyses rely on large Missing Transverse Energy ( $p_{\mathrm{T}}^{\text {miss }}$ ) balancing against "visible" (X) objects (jets, boosted-jets, b-jets, photons, charged leptons), where $\Delta \phi_{p_{\mathrm{T}}^{\text {miss }}, X} \sim \pi$ and $p_{\mathrm{T}}^{\text {miss }} \sim p_{\mathrm{T}}^{X}$. Therefore, understanding $p_{\mathrm{T}}^{\text {miss }}$ is a critical component. As an example, the $p_{\mathrm{T}}^{\text {miss }}$ distribution in $\mathrm{Z} \rightarrow \ell \ell$ events before and after applying some data cleaning is shown in Fig. 2.

Another key ingredient is the selection of the interesting events via a set of online trigger paths. Most multijet analyses rely on $p_{\mathrm{T}}^{\text {miss }}$ triggers, which are designed in similar way as the offline selection, but with more basic and faster algorithms. In particular, the efficiency turn-on reaches $\sim 100 \%$ at $p_{\mathrm{T}}^{\text {miss }} \sim 250 \mathrm{GeV}$ for an online trigger requiring $p_{\mathrm{T}}^{\text {miss }}>150 \mathrm{GeV}$, where the online thresholds are dictated by the rate of the trigger paths.

\section{Analyses}

A large set of analyses are performed using the CMS data with the goal of searching for DM in all possible and feasible final states: 


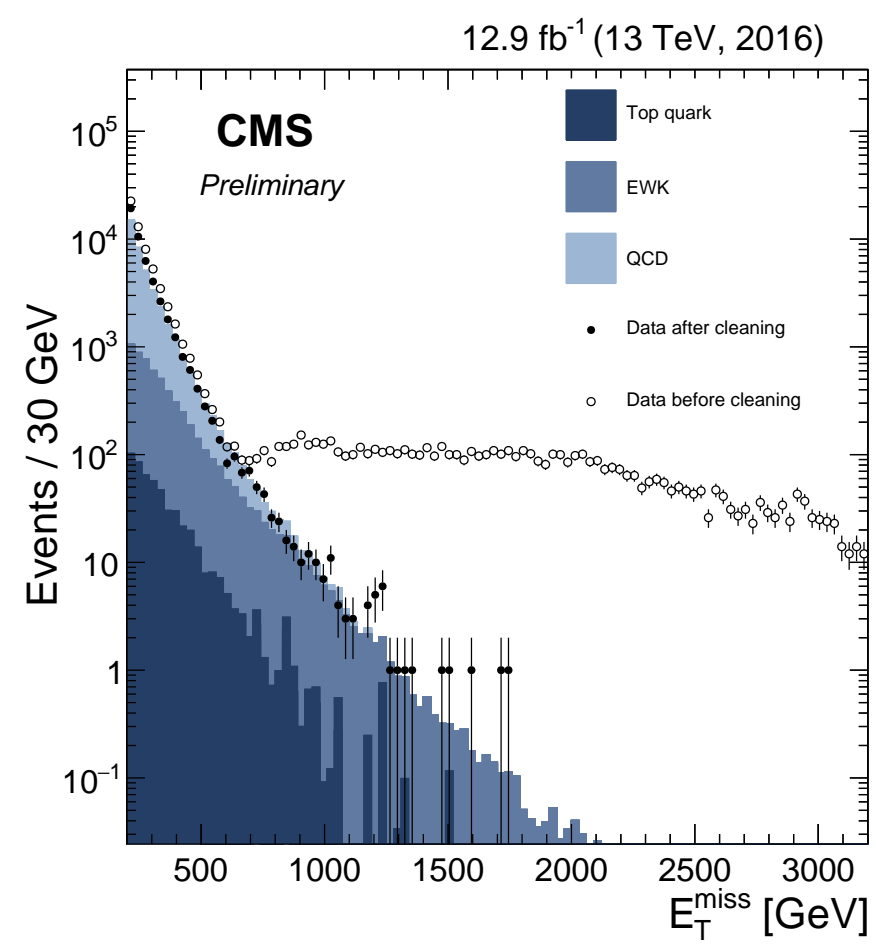

Figure 2: The $p_{\mathrm{T}}^{\text {miss }}$ distribution in $\mathrm{Z} \rightarrow \ell \ell$ events before and after applying some data cleaning [2].

- $p_{\mathrm{T}}^{\mathrm{miss}}+\mathrm{X}$

- mono-X

* Mono-jet: one or more jets

* Mono- $\mathrm{V}(q q)$ : a single boosted jet compatible with an hadronic $\mathrm{W} / \mathrm{Z}$ boson decay

* Mono-photon: a single energetic and isolated photon

* Mono-Z $(\ell \ell)$ : a single $\mathrm{Z} \rightarrow \ell \ell$ boson decay

* Mono-top: a single boosted top

* Vector Boson Fusion: a pair of jets compatible with such production, mostly on $\mathrm{H} \rightarrow$ invisible models

* Mono-H: a SM Higgs boson decaying to $b \bar{b}, \gamma \gamma, \tau \tau$, WW...

- DM + Heavy Flavor

* $\mathrm{t} \overline{\mathrm{t}}:$ a top-quark pair in all possible decaying final states

* $b \bar{b}$ : a b-quark pair

- Di-jet

$-\chi \rightarrow j j$, bump hunting or angular analyses

- Multijet $+p_{\mathrm{T}}^{\text {miss }}$ SUSY reinterpretations 
- Long-lived + DM decays

The talk at the conference was mostly dedicated to the so-called mono-X analyses, but for clarity other type analyses are also mentioned above.

\section{Results}

A summary of the $95 \%$ confidence level (CL) observed and expected exclusion regions as a function of the mediator mass $\left(m_{M e d}\right)$ and the DM mass $\left(m_{D M}\right)$ in the $m_{M e d}-m_{D M}$ plane for different $p_{\mathrm{T}}^{\text {miss }}$ based DM searches from CMS in the lepto-phobic Axial-vector and Vector models [3] is shown in Fig. 3. Comparisons of CMS results to the spin-dependent (SD) and spin-independent (SI) cases in the $m_{D M}-\sigma_{S D}$ and $m_{D M}-\sigma_{S I}$ planes, using upper limits at the $90 \% \mathrm{CL}$, with other experiments are shown in Fig. 4. Documentation for all individual analyses can be found on [4].
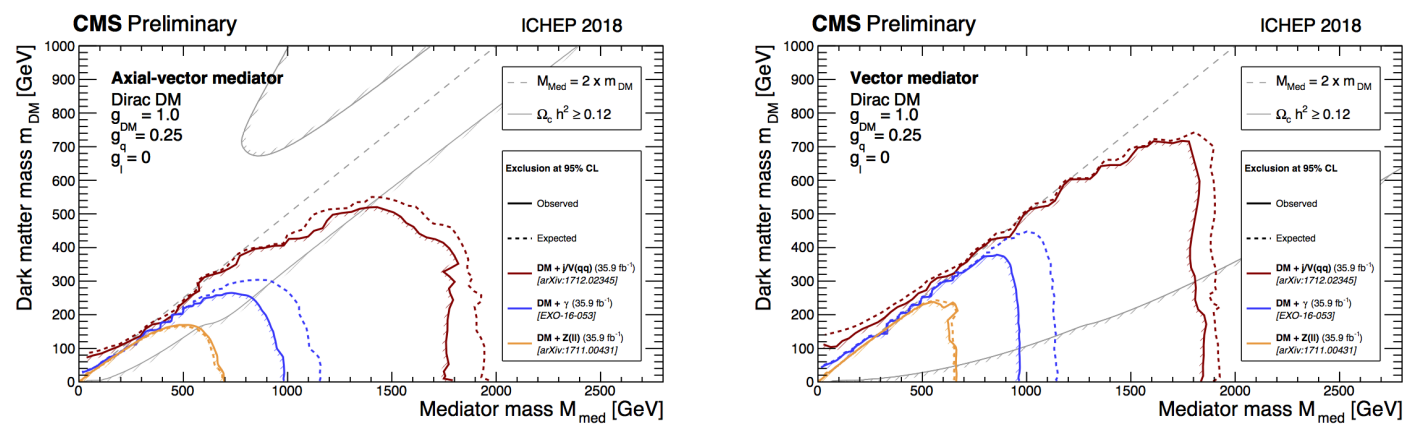

Figure 3: 95\% CL observed and expected exclusion regions in $m_{M e d}-m_{D M}$ plane for different $p_{\mathrm{T}}^{\text {miss }}$ based DM searches from CMS in the lepto-phobic Axial-vector (left) and Vector (right) models. The exclusions are computed for a universal quark coupling of $g_{q}=0.25$ and for a DM coupling of $g_{D M}=1.0$ [4].
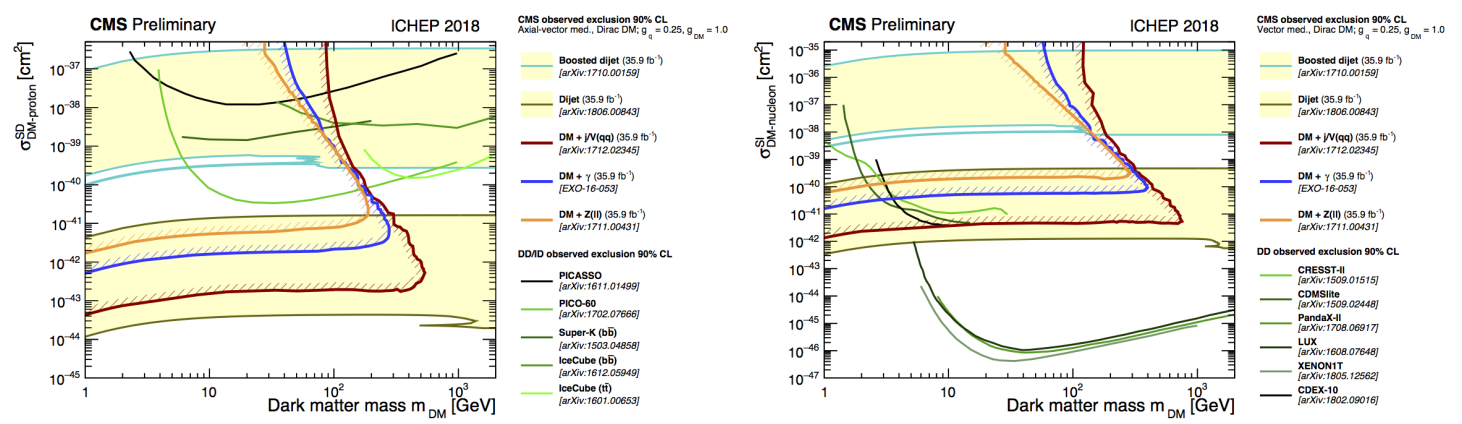

Figure 4: Comparison of CMS results in the $m_{D M}-\sigma_{S D}$ (left) and $m_{D M}-\sigma_{S I}$ (right) planes, using upper limits at the $90 \% \mathrm{CL}$, with other experiments. The CMS contour in the SD plane is for an Axial-vector mediator, Dirac DM and couplings $g_{q}=0.25$ and $g_{D M}=1.0$; while the CMS contour in the SI plane is for a Vector mediator, Dirac DM and couplings $g_{q}=0.25$ and $g_{D M}=1.0$ [4].

An specific case is the search for invisible Higgs boson decays. It exists in the SM, but extremely rare, with $B(\mathrm{H} \rightarrow \mathrm{ZZ} \rightarrow 4 v) \sim 0.1 \%$. Therefore, the observation of a large rate would 
be a sign of physics beyond the SM, and it is the most extensive set of rare Higgs boson decays analyses by far [5]. The observed and expected 95\% CL upper limits on $B(\mathrm{H} \rightarrow$ invisible $)) / \sigma_{S M}$ for all individual categories, as well as their combination, assuming a SM Higgs boson with a mass of $125 \mathrm{GeV}$ are shown in Fig. 5.

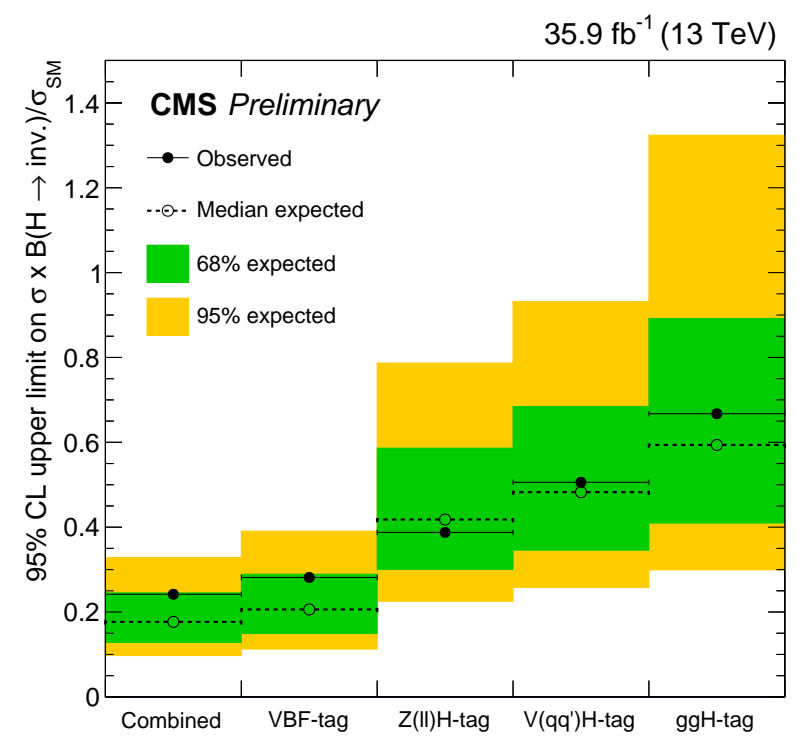

Figure 5: Observed and expected $95 \% \mathrm{CL}$ upper limits on $B R(\mathrm{H} \rightarrow$ invisible $)) / \sigma_{S M}$ for all individual categories, as well as their combination, assuming a SM Higgs boson with a mass of $125 \mathrm{GeV}$ [5].

\section{Summary}

This conference report has briefly reviewed the status of the DM searches the CMS experiment with the data collected in 2016 at $\sqrt{s}=13 \mathrm{TeV}$ using a relatively large number of different final states. In particular, the talk was giving emphasis in the so-called mono-X final states, where a large number of analyses have been performed in CMS. In all cases, no significant excess of events has been found over the background prediction.

\section{References}

[1] CMS Collaboration, 'The CMS experiment at the CERN LHC, JINST 3:S08004,2008.

[2] CMS Collaboration, CMS Physics Analysis Summary, CMS-PAS-JME-16-004, "Performance of missing energy reconstruction in $13 \mathrm{TeV}$ pp collision data using the CMS detector", http://cds.cern.ch/record/2205284.

[3] See https://twiki.cern.ch/twiki/bin/view/CMSPublic/SummaryPlotsEXO13TeV.

[4] See http://cms-results.web.cern.ch/cms-results/public-results/publications/EXO/index.html.

[5] CMS Collaboration, CMS Physics Analysis Summary, CMS-PAS-HIG-17-023, "Search for invisible decays of the Higgs boson produced through vector boson fusion at $\sqrt{s}=13 \mathrm{TeV}$ ", http://cds.cern.ch/record/2308434. 